\title{
LUNAR MOTION: THEORY AND OBSERVATIONS
}

\author{
JEAN CHAPRONT AND MICHELLE CHAPRONT-TOUZÉ \\ Bureau des Longitudes \\ Paris, France
}

\begin{abstract}
Results of several fits of the lunar theory ELP 2000-82B and of Moons' theory of libration are presented. The theories are fitted both to JPL numerical integrations and to LLR observations.
\end{abstract}

\section{Introduction}

Several fits of the semi-analytical theory ELP 2000-82B for the lunar orbital motion and of Moons' analytical theory of libration have been performed recently. On one hand, the analytical solutions have been fitted to the JPL numerical integrations DE245 and DE403 (Standish et al., 1995) on the time span [1900 - 2010] for the orbital motion, and to DE245 over the time span $[1750-2050]$ for the libration. We have obtained in these fits values of the orbital parameters of the Moon and of the Earth-Moon barycenter, values of bias parameters (observed corrections to the half secular acceleration of the longitude and to the mean motions of perigee and node), values of the so called free libration parameters, and position of the mean ecliptic of J2000.0 with respect to the equators and equinoxes of DE245 and DE403.

On the other hand, the analytical solutions have been fitted to a set of LLR observations covering the time span [January 1972 - October 1993]. Values of the same parameters have been obtained, the position of the mean ecliptic of J2000.0 being referred this time to the mean CEP of J2000.0. A correction to the IAU 1976 value of the precession constant has also been obtained. In this paper, the adopted time scale is TDB.

\section{Comparison of ELP 2000-82B to DE245 and DE403}

ELP 2000-82B consists of the series of ELP 2000-82 (Chapront-Touzé and Chapront, 1983) and of the angular mean elements of ELP 2000-85 (Cha- 
pront-Touzé and Chapront, 1988), i.e. time polynomials of degree 4. Furthermore, for the fits described in this paper, several improvements have been brought to the original ELP 2000-82B:

- DE245 values of the geocentric constant of gravitation and ratio of the lunar and terrestrial masses have been introduced.

- The perturbations due to the Earth figure and those due to the lunar figure have been computed again with the values of the gravitational parameters of DE245.

- The tidal perturbations have been computed with a new model separating diurnal and semi-diurnal tides on the Earth and taking into account tides on the Moon; the values of the physical constants involved are those of DE245.

It results from these modifications:

- a correction of -3.80 meters to the distance computed with the DE200 values of the geocentric constant of gravitation and ratio of the lunar and terrestrial masses $(-18.71$ meters to the distance computed with the IAU 1976 values) ;

- corrections of 0.0556 and $-0.1604 \mathrm{arcsec} / \mathrm{cy}$ respectively to the mean motions of perigee and node ;

- a correction of $-0.8652 \mathrm{arcsec} / \mathrm{cy}^{2}$ to the coefficient of the quadratic term of the mean mean longitude, the resulting new value of the tidal part of this coefficient being $-12.8125 \mathrm{arcsec} / \mathrm{cy}^{2}$ (the coefficient of the quadratic term of the mean mean longitude is the half secular acceleration, i.e. the half second derivative of the mean mean longitude with respect to time, in $\mathrm{J} 2000.0$ ) ;

- a secular correction of 0.25 meter/cy to the distance ;

- a correction of $-0^{\prime \prime} 00059 \sin \left(D+l^{\prime}-F+192^{\circ}\right)$ to the longitude, due to the new model of tidal perturbations.

We denote here as "DE200 values" the values of the orbital parameters of the Moon and of the Earth-Moon barycenter fitted to DE200 and given in Table 9 of (Chapront-Touzé and Chapront, 1983).

We give in columns (1) of Table 1 the results of the fit of the improved ELP 2000-82B to DE245, under the form of corrections to the DE200 values for orbital parameters. For parameters fixing the position of the ecliptic and bias parameters, the complete values are given. Table 1 gives also the minima and maxima of the post fit residuals. The lunar orbital parameters are $W_{1}^{(0)}, W_{2}^{(0)}, W_{3}^{(0)}$ the values in $\mathrm{J} 2000.0$ of the mean mean longitude $W_{1}$, mean longitude of perigee $W_{2}$ and mean longitude of node $W_{3}$ referred to a mean inertial equinox of J2000.0 $\gamma_{n}^{I}$ defined below, the sidereal mean motion $\nu$, the inclination constant $\Gamma$ (the half coefficient of $\sin F$ in the latitude), and the eccentricity constant $E$ (the half coefficient of $\sin l$ in the 
longitude). The orbital parameters of the Earth-Moon barycenter are the values in J2000.0 of the mean mean longitude $T$ and of the mean longitude of perihelion $\varpi^{\prime}$, the sidereal mean motion $n^{\prime}$, and the eccentricity $e^{\prime}$. The parameters fixing the position of the mean ecliptic of J2000.0, inertial ecliptic in the sense of (Standish, 1981), with respect to the equator and equinox of the numerical integration DEn involved in the fit are the right ascension $\varphi$ of $\gamma_{n}^{I}$, the ascending node of the ecliptic on the equator, and the inclination $\varepsilon$ of the ecliptic. $\varphi$ is reckonned in the equator of DEn from the equinox of $\mathrm{DEn}$. The bias parameters are fitted corrections $\Delta W_{1}^{(2)}, \Delta W_{2}^{(1)}$, $\Delta W_{3}^{(1)}$, to the theoretical values of the coefficients of the quadratic term of $W_{1}$ and of the linear terms of $W_{2}$ and $W_{3}$, the theoretical values being computed for the values of the orbital parameters obtained in the present fit. The columns $\sigma$ give the formal errors of the least square adjustment.

It was also shown in our paper of 1983 that a precise lunar solution might be obtained by adding to ELP 2000-82 numerical complements built from the residuals of the fit of the analytical solution to a numerical integration. In this perspective, numerical complements to the improved analytical solution ELP 2000-82B have been built by approximating the residuals of the fit to DE245 by Chebychev polynomials. The numerical complements involve the periodic terms which are missing in our analytical solution, mainly in planetary perturbations series, and the corrections due to the update of planetary masses. The update of the gravitational and tidal parameters of the Earth and Moon have been introduced in the series. We may consider, within a good approximation, that numerical complements do not depend of the corrections for the orbital parameters of the Moon and of the Earth-Moon barycenter, of the parameters fixing the position of the ecliptic, and of the periodic terms induced by tidal forces as far as our model is supposed to be close to that of DE245. Besides, they do not involve corrections to the theoretical mean motions of perigee and node, and secular acceleration because such quantities are taken off by the fit. Numerical complements could have been computed with the same accuracy from the residuals of the fit of ELP 2000-82 to any other numerical integration as far as the latter realizes a mathematically precise solution of the differential equations with the same model of forces as ELP and update values of the planetary masses. The initial conditions of such an integration should be in the vicinity of those which represent the real motion but not necessarily the best ones.

The improved analytical solution ELP 2000-82B plus those numerical complements have been fitted to the JPL numerical integration DE403. The results are given in columns (2) of Table 1. The orbital parameters are quoted as corrections to the values of columns (1) (DE245 values). For the other parameters the complete values are given. 
TABLE 1. Results of the fits of the improved ELP 2000-82B to DE245 (1), and of the improved ELP 2000-82B plus numerical complements to DE403 (2) and to LLR observations (3). Units: arcsecond, century, and meter.

\begin{tabular}{|c|c|c|c|c|c|c|}
\hline \multirow[b]{2}{*}{ Variable } & \multicolumn{2}{|c|}{ (1) } & \multicolumn{2}{|c|}{$(2)$} & \multicolumn{2}{|c|}{ (3) } \\
\hline & value & $\sigma$ & value & $\sigma$ & value & $\sigma$ \\
\hline \multicolumn{7}{|c|}{ Orbital parameters } \\
\hline & \multicolumn{2}{|c|}{$\begin{array}{l}\text { Corrections to } \\
\text { DE200 values }\end{array}$} & \multicolumn{2}{|c|}{$\begin{array}{c}\text { Corrections to } \\
\text { values (1) }\end{array}$} & \multicolumn{2}{|c|}{$\begin{array}{c}\text { Corrections to } \\
\text { values (1) }\end{array}$} \\
\hline$W_{1}^{(0)}$ & -0.12089 & 0.00017 & 0.04002 & 0.00001 & -0.00511 & 0.00268 \\
\hline$W_{2}^{(0)}$ & -0.06736 & 0.00089 & 0.04002 & 0.00003 & -0.00786 & 0.00279 \\
\hline$W_{3}^{(0)}$ & -0.09950 & 0.00122 & 0.03871 & 0.00004 & -0.04545 & 0.01246 \\
\hline$\nu$ & -0.37990 & 0.00046 & 0.00010 & 0.00002 & -0.03888 & 0.00798 \\
\hline$\Gamma$ & 0.00121 & 0.00003 & 0.00010 & 0.00000 & -0.00045 & 0.00027 \\
\hline$E$ & 0.00013 & 0.00003 & 0.00001 & 0.00000 & 0.00003 & 0.00000 \\
\hline$T^{(0)}$ & -0.07713 & 0.00194 & 0.04007 & 0.00007 & -0.00870 & 0.00275 \\
\hline$\varpi^{\prime(0)}$ & -0.05626 & 0.01678 & 0.03975 & 0.00062 & 0.00216 & 0.00469 \\
\hline$n^{\prime}$ & 0.03199 & 0.00348 & -0.00510 & 0.00013 & -0.06388 & 0.00569 \\
\hline$e^{\prime}$ & 0.00000 & 0.00028 & 0.00000 & 0.00001 & 0.00000 & 0.00006 \\
\hline \multicolumn{7}{|c|}{ Ecliptic parameters } \\
\hline$\varphi$ & -0.07355 & 0.00016 & -0.05294 & 0.00001 & -0.01574 & 0.00286 \\
\hline$\varepsilon-23^{\circ} 26^{\prime} 21^{\prime \prime}$ & 0.40580 & 0.00006 & 0.40928 & 0.00000 & 0.40831 & 0.00060 \\
\hline \multicolumn{7}{|c|}{ Bias parameters } \\
\hline$\Delta W_{1}^{(2)}$ & -0.04608 & 0.00049 & -0.02371 & 0.00002 & -0.00184 & 0.01660 \\
\hline$\Delta W_{2}^{(1)}$ & 0.05217 & 0.00327 & 0.05172 & 0.00012 & -0.00129 & 0.01082 \\
\hline$\Delta W_{3}^{(1)}$ & -0.01759 & 0.00228 & -0.02455 & 0.00008 & -0.49669 & 0.06789 \\
\hline \multicolumn{7}{|c|}{ Minima and maxima of the residuals } \\
\hline Longitude & {$[-0.0120$} & $0.01110]$ & {$[-0.00008$} & $0.00008]$ & & \\
\hline Latitude & {$[-0.0047$} & $0.00476]$ & {$[-0.0002]$} & $0.00021]$ & & \\
\hline Distance & {$[-12.0$} & $14.24]$ & {$[-0.17$} & $-0.13]$ & & \\
\hline
\end{tabular}

The introduction of numerical complements makes the formal errors and the residuals decrease considerably. The fit can now be realized at the centimeter level. The residuals in longitude represented in columns (2) of Table 1 are partially imputable to differences of values for the physical parameters involved in the tidal perturbations between DE245 and DE403. They become smaller than 0.00003 in magnitude if we replace in ELP 2000-82B the series of the tidal perturbations computed with DE245 values by those computed with DE403 values (the model of forces is the same). This difference of constants induces also a difference of $0.02272 \mathrm{arcsec} / \mathrm{cy}^{2}$ between the tidal parts of the coefficients of the quadratic term of the longitude in DE245 and DE403 which accounts satisfactorily for the differences between the values of $\Delta W_{1}^{(2)}$ in columns (1) and (2). 
Similarly, the difference between the values of the geocentric constant of gravitation in the two numerical integrations induces a difference of -0.18 meter in the distance which is visible in our residuals. At last, the residuals in latitude can also be reduced below 0." 00003 with a small secular variation of $\varphi$ and $\varepsilon$ but this phenomenon is not yet explained.

The corrections to the DE200 values of the angular orbital parameters given in columns (1) are partially due to the separation between the origins $\gamma_{200}^{I}$ and $\gamma_{245}^{I}$. We have assumed that the DE200 constants yield a precise lunar mean mean longitude referred to $\gamma_{200}^{I}$ in 1975.0, the mean epoch of the LLR observations involved in DE200. Then, corrections of columns (1) allow to compute, for the same epoch, the difference " $W_{1}$ referred to $\gamma_{245}^{I}-W_{1}$ referred to $\gamma_{200}^{I}$ " which is the arc $\gamma_{245}^{I} \gamma_{200}^{I}$ measured in the mean inertial ecliptic of J2000.0. We have obtained $\gamma_{245}^{I} \gamma_{200}^{I}=-0$. $08158 \pm$ 0.00060 . Compatible values have been obtained from the other angular elements except the node. Similarly, columns (2) give $\gamma_{403}^{I} \gamma_{245}^{I}=0.04073 \pm$ 0.00003 .

\section{Comparison of Moons' Theory of Physical Libration to DE245}

Moons' analytical theory of physical libration (Moons, 1981, 1982, and 1984) provides analytical expressions for the variables $p_{1}, p_{2}$ and $\tau . p_{1}$ and $p_{2}$ are the components of the unit vector pointing towards the mean pole of the ecliptic of date on the two lunar equatorial principal axes of inertia; $\tau$ is the libration in longitude. The series depending on the free libration involve three litteral arguments $p, q, r$, whose frequencies are given by the theory in function of the gravitational parameters of the Moon. Their coefficients involve the gravitational parameters of the Moon and three litteral constants denoted as $\sqrt{2 P}, \sqrt{2 Q}, \sqrt{2 R}$.

For the fit described in this paper, we have completed Moons' forced libration by perturbations due to tidal effect and by direct perturbations induced by Earth figure (spin-spin perturbations). These perturbations have been computed under the form of semi-analytical series and will be published later. The fit has been performed in the following way. The Euler angles provided by DE245 have been converted to variables $p_{1}, p_{2}, \tau$ by using the parameters $\phi$ and $\varepsilon$ and the mean mean longitude $W_{1}$ fitted to DE245 (sect. 2).

Theoretical values of $p_{1}, p_{2}, \tau$ have been obtained by substituting both DE245 values of the gravitational and tidal parameters, and lunar arguments fitted to DE245 in the forced part of the theory. The differences have been treated by frequency analysis over a time span of three centuries from 1750 to 2050 . The terms of the series which depend on the free libration have been identified to peaks of the spectrum. The identification 
TABLE 2. Results of the fits of the improved Moons' libration theory to DE245 (1) and to LLR observations (2).

\begin{tabular}{|c|c|c|c|c|}
\hline \multirow[b]{2}{*}{ Variable } & \multirow{2}{*}{$\begin{array}{c}(1) \\
\text { value }\end{array}$} & \multicolumn{2}{|c|}{ (2) } & \multirow[b]{2}{*}{ unit } \\
\hline & & value & $\sigma$ & \\
\hline \multirow{2}{*}{\multicolumn{5}{|c|}{$\begin{array}{l}\text { Values of the fitted parameters } \\
\text { corrections to } \\
\text { values }(1)\end{array}$}} \\
\hline & & & & \\
\hline$p^{(0)}$ & 224.2791 & 1.74111 & 0.11376 & $\operatorname{deg}$ \\
\hline$q^{(0)}$ & 161.6206 & -0.50271 & 0.04991 & $\operatorname{deg}$ \\
\hline$r^{(0)}$ & 124.3579 & -1.19762 & 8.44297 & $\operatorname{deg}$ \\
\hline$p^{(1)}$ & 44820417 & & & $\operatorname{arcsec} / c y$ \\
\hline$q^{(1)}$ & 1736493 & & & $\operatorname{arcsec} / c y$ \\
\hline$\sqrt{2 P}$ & 0.2922 & 0.00948 & 0.00053 & \\
\hline$\sqrt{2 Q}$ & 5.1968 & 0.04680 & 0.01379 & \\
\hline$\sqrt{2 R}$ & 0.0207 & 0.01208 & 0.00283 & \\
\hline \multicolumn{5}{|c|}{ Minima and maxima of the residuals over $[1750-2050]$} \\
\hline$p_{1}$ & {$[-0.25 ; 0.25]$} & & & $\operatorname{arcsec}$ \\
\hline$p_{2}$ & {$[-0.25 ; 0.25]$} & & & $\operatorname{arcsec}$ \\
\hline$\tau$ & {$[-0.60 ; 1.00]$} & & & $\operatorname{arcsec}$ \\
\hline \multicolumn{5}{|c|}{ Minima and maxima of the residuals over $[1969-2000]$} \\
\hline$p_{1}$ & {$[-0.08 ; 0.08]$} & & & $\operatorname{arcsec}$ \\
\hline$p_{2}$ & {$[-0.10 ; 0.08]$} & & & $\operatorname{arcsec}$ \\
\hline$\tau$ & {$[-0.20 ; 0.20]$} & & & $\operatorname{arcsec}$ \\
\hline
\end{tabular}

of the terms with argument $q$ in $p_{1}$ and $p_{2}$ and argument $p$ in $\tau$ yields fitted values of the frequencies $p^{(1)}$ and $q^{(1)}$ of these arguments, of their values $p^{(0)}$ and $q^{(0)}$ in $\mathrm{J} 2000.0$, and of $\sqrt{2 P}$ and $\sqrt{2 Q}$. The identification of the terms with argument $F+r$ in $p_{1}$ and $p_{2}$ yields fitted values of $r^{(0)}$ and of $\sqrt{2 R}$. Column (1) of Table 2 gives the so obtained results. The leading parts (coefficients greater than 0.005 ) of the series depending on the free libration in Moons' theory after substitution of the physical parameters of $\mathrm{DE} 245$ and of the fitted values of $\sqrt{2 P}, \sqrt{2 Q}$, and $\sqrt{2 R}$ are:

$$
\begin{aligned}
& p_{1}^{F}=-3^{\prime \prime} \cdot 298 \sin (q-0.01)+0{ }^{\prime \prime} 034 \sin (q-l)+0.026 \sin (p-F) \\
& +0.023 \sin (p+F)-0 . \prime 022 \sin (q+l)+0 .{ }^{\prime \prime} 021 \sin (F+r) \\
& p_{2}^{F}=8^{\prime \prime} .163 \cos (q+0.01)-0 . \prime 035 \cos (q-l)-0.026 \cos (p-F) \\
& +0^{\prime \prime} 023 \cos (p+F)-0.022 \cos (q+l)+0.021 \cos (F+r) \\
& \tau^{F}=1^{\prime \prime} 817 \sin p+0^{\prime \prime} 088 \sin \left(q+2 F-2 l+36^{\circ}\right)+0.077 \sin (q+F) \\
& +0.069 \sin (q+F-l)-0.033 \sin (q-F)+0.015 \sin (q-F+l) \text {; }
\end{aligned}
$$


The determination of an accurate fitted value of the frequency $r^{(1)}$ of $r$ (theoretical period: 24.1579 years) is not possible in that way since, in $p_{1}$ and $p_{2}, r$ is added to $F$ whose period is much smaller, and in $\tau r$ has a too small coefficient. Furthermore, the values of $p^{(0)}, q^{(0)}, r^{(0)}, \sqrt{2 P}$, $\sqrt{2 Q}$ and $\sqrt{2 R}$ obtained here may involve spurious contributions due to missing or unprecise terms with frequencies close to those of $p, q$, and $F+r$ in the series of the forced libration. This could explain the discrepancies between the fitted periods of $p$ (2.8915 years) and $q$ ( 74.6332 years) and the theoretical ones, respectively 2.8449 and 75.4060 years.

The minima and maxima of the differences DE245 - "improved Moons' analytical solution for forced and free libration plus constants of Table 2 (1)" are given in column (1) of Table 2 .

\section{Comparison of Improved ELP 2000-82B and Moons' Libration Theory to LLR Observations}

We give in columns (3) of Table 1 and in columns (2) of Table 2 the values of the same variables obtained by fitting the improved ELP 2000-82B plus numerical complements and the improved Moons' libration theory directly to LLR observations from January 1972 to October 1993. A detailed description of this fit will be given elsewhere. We mention only that here $\gamma_{n}^{I}$ of sect. 2 is replaced by $\gamma_{E}^{I}$ the ascending node of the inertial mean ecliptic of $\mathrm{J} 2000.0$ on the "mean celestial ephemeris equator of J2000.0" (the equator of the mean CEP of J2000.0), $\varphi$ being the right ascension of $\gamma_{E}^{I}$ reckoned from "the mean celestial ephemeris equinox of J2000.0" compatible with UT1 provided by IERS. Similarly to sect. 2, columns (3) of Table 1 yield $\gamma_{E}^{I} \gamma_{245}^{I}=0 ! 00332 \pm 0.00470$

\section{Conclusion}

The addition of numerical complements to ELP 2000-82B yields a mixed orbital solution which has both the advantages of an analytical solution (i.e. the integration constants, physical constants, and models of forces can be easily changed) and of a numerical one (precision at the centimeter level).

For orbital elements, the results of the fit of this solution and of the improved Moons' solution for the libration to LLR observations are in good agreement with the improvements of DE245 and DE403 with respect to DE200 except for the solar mean motion.

The same fit yields $-12.81 \pm 0.02 \mathrm{arcsec} / \mathrm{cy}^{2}$ for the tidal part of the coefficient of the quadratic term of the longitude and $-0.32 \pm 0.02 \mathrm{arcsec} / \mathrm{cy}$ for the precession constant, in good agreement with modern determinations.

From the values of the ecliptic parameters given in Table 1 and from the arc separating the various $\gamma^{I}$ given in sect. 2 and sect. 4 , it is easy to 
compute the three angles $\theta_{1}^{k n}, \theta_{2}^{k n}, \theta_{3}^{k n}$ defined by

$$
x_{k}=R_{1}\left(\theta_{1}^{k n}\right) R_{2}\left(\theta_{2}^{k n}\right) R_{3}\left(\theta_{3}^{k n}\right) x_{n}
$$

where $x_{k}$ is the position vector referred to the J2000.0 equator and equinox $k$ and $R_{i}$ the rotation matrix around axis $i$. We find $\theta_{1}^{200,403}=0.45 \pm 0.06$ mas, $\theta_{2}^{200,403}=16.25 \pm 0.25$ mas, $\theta_{3}^{200,403}=2.03 \pm 0.75$ mas, and $\theta_{1}^{E, 403}=$ $0.97 \pm 0.60$ mas, $\theta_{2}^{E, 403}=-14.88 \pm 1.90$ mas, $\theta_{3}^{E, 403}=-2.88 \pm 7.20$ mas. DE403 being referred to the IERS celestial reference frame, the first set is in good agreement with the vector $A_{I E R S}^{D E 200}$ given by (Folkner et al., 1994), and the first two elements of the second set are in good agreement with the coordinates of the mean CEP of J2000.0 in the IERS celestial reference frame given by the same authors.

Values of the "free libration parameters" have been determined both by comparison to DE245 and to LLR observations. The agreement between the two sets of values is satisfactory for $(P, p)$ and $(Q, q)$, and less good for $(R, r)$.

Acknowledgements. The authors are very grateful to Christian Veillet for providing the files of LLR observations, Gérard Francou for his programs of ephemerides, and Michèle Moons for her files of libration.

\section{References}

Chapront-Touzé, M. and Chapront, J.: 1983, "The Lunar ephemeris ELP 2000", Astron. Astrophys. 124, 50.

Chapront-Touzé, M. and Chapront, J.: 1988, "ELP 2000-85: A semi-analytical Lunar ephemeris adequate for historical times", Astron. Astrophys. 190, 342.

Folkner, W.M., Charlot, P., Finger, M.H., Williams, J.G., Sovers, O.J., Newhall, X X, and Standish, E.M.: 1994, "Determination of the extragalactic-planetary frame tie from joint analysis of radio interferometric and Lunar laser ranging measurement", Astron. Astrophys. 287, 279.

Moons, M.: 1981, Libration physique de la Lune, Thesis, Facultés Universitaires de Namur.

Moons, M.: 1982, "Analytical theory of libration of the Moon", The Moon and the Planets 27, 257.

Moons, M.: 1984, "Planetary perturbations on the libration of the Moon", Celest. Mech. 34, 263.

Standish, E.M.: 1981, "Two differing definitions of the dynamical equinox and the mean obliquity", Astron. Astrophys. 101, L17.

Standish, E.M., Newhall, X X, Williams, J.G., and Folkner, W.M.: 1995, "JPL planetary and Lunar ephemerides, DE 403/LE403", JPL IOM 314.10-127. 